\title{
Natural history of asymptomatic renal artery pseudoaneurysm after robot-assisted partial nephrectomy
}

\author{
Tatsuya Takayama ${ }^{1} \wedge$, Akifumi Fujita ${ }^{2}$, Toru Sugihara ${ }^{1}$, Akira Fujisaki ${ }^{1}$, Masahiro Yamazaki ${ }^{1}$, \\ Tomohiro Kameda ${ }^{1}$, Jun Kamei ${ }^{1}$, Satoshi Ando ${ }^{1}$, Shinsuke Kurokawa ${ }^{1}$, Tetsuya Fujimura ${ }^{1}$ \\ ${ }^{1}$ Department of Urology, Jichi Medical University, Shimotsuke, Tochigi, Japan; ${ }^{2}$ Department of Radiology, Jichi Medical University, Shimotsuke, \\ Tochigi, Japan \\ Contributions: (I) Conception and design: T Takayama; (II) Administrative support: A Fujita, T Sugihara; (III) Provision of study materials or patients: \\ T Takayama, A Fujisaki, M Yamazaki, T Kameda, J Kamei, S Ando, S Kurokawa; (IV) Collection and assembly of data: T Takayama, A Fujita, \\ A Fujisaki, M Yamazaki, T Kameda, J Kamei, S Ando, S Kurokawa; (V) Data analysis and interpretation: T Takayama, A Fujita, T Sugihara, T \\ Fujimura; (VI) Manuscript writing: All authors; (VII) Final approval of manuscript: All authors. \\ Correspondence to: Tatsuya Takayama, MD, PhD. 3311-1 Yakushiji Shimotsuke, Tochigi 329-0498, Japan. Email: ttakayam@jichi.ac.jp.
}

Background: We assessed the natural history of renal artery pseudoaneurysm (RAP) after robot-assisted partial nephrectomy (RAPN).

Methods: From May 2016 to September 2020, 106 patients underwent RAPN for renal tumors at our institution. Among 100 patients, excluding 6 who were ineligible for contrast-enhanced computed tomography (CE-CT), 4 underwent renal artery selective embolization (RAE), of which 2 cases were emergency RAE within 7 days after RAPN and the other 2 were prophylactic RAE 8 or more days after RAPN. In 98 patients examined for the clinical course of asymptomatic RAP managed by surveillance, excluding the 2 who underwent emergency RAE, routine CE-CT was performed at 7 days, 1 month and 3 months after RAPN. Factors influencing the occurrence of RAP among these 98 patients, including the 2 who underwent emergency RAE and excluding the 2 who underwent prophylactic RAE, were analyzed by logistic regression analysis.

Results: Median [interquartile range (IOR), range] observation period, age, radiographic tumor size, and maximum diameter of RAP were 20.8 (23.9, 3.0-57.6) months, 63 (18, 22-84) years, $23(11,9-48) \mathrm{mm}$, and $6.6(5.2,3.0-16.0) \mathrm{mm}$, respectively. CE-CT detected 28 RAPs in 23 (23.0\%) of 100 patients by 7 days after RAPN and routine CE-CT detected 25 RAPs in 21 (21.4\%) of 98 patients excluding 2 who underwent emergency RAE at 7 days after RAPN. RAP was diagnosed by routine CE-CT in 21 (21.4\%), 1 (1.0\%), and $0(0 \%)$ patients at 7 days, 1 month, and 3 months after RAPN, respectively. In univariate analysis, age [odds ratio (OR) 0.144: 69-84 vs. 22-56 years old, $\mathrm{P}=0.0179$ ], R.E.N.A.L [radius (tumor size as maximal diameter), exophytic/endophytic properties of tumor, nearness of tumor deepest portion to collecting system or sinus, anterior/posterior descriptor and location relative to polar line] nephrometry score (OR 1.374, $\mathrm{P}=0.0382$ ), warm ischemic time (OR 1.085, $\mathrm{P}=0.0393)$, and renorrhaphy time (OR 1.055, $\mathrm{P}=0.0408$ ) were significantly associated with the occurrence of RAP. In multivariate analysis, only age (OR $0.124, \mathrm{P}=0.0148)$ was a significant factor.

Conclusions: Asymptomatic RAP up to $15 \mathrm{~mm}$ in diameter resolved spontaneously 3 months after RAPN. Young age (under 56 years) may be a factor in the development of RAP.

Keywords: Kidney neoplasms; comorbidity; robotics; age; pseudoaneurysm

Submitted Apr 29, 2021. Accepted for publication Jul 15, 2021.

doi: $10.21037 /$ tau-21-384

View this article at: https://dx.doi.org/10.21037/tau-21-384

^ ORCID: 0000-0003-3360-7688. 


\section{Introduction}

Partial nephrectomy $(\mathrm{PN})$ has been successful in reducing mortality in patients with small renal cancer through both the achievement of promising oncological outcomes and reducing cardiac events associated with the protection of renal function, which is still controversial. $\mathrm{PN}$ has now replaced radical nephrectomy as the gold standard treatment for these cancers $(1,2)$. The procedure initially evolved from open PN (OPN) to laparoscopic PN (LPN), while in recent years, robot-assisted PN (RAPN) has become more common. Progress towards less invasive and safer surgery has been steady, supported by technological development and ingenuity such as in the use of $3 \mathrm{D}$ models (3-5). One of the most stressful and life-threatening complications for patients who undergo $\mathrm{PN}$ is renal artery pseudoaneurysm (RAP) (6). Previous studies have reported incidence rates of RAP after PN of $1-5 \%$ (6). The frequency of RAP increased from $1.00 \%$ with OPN to $1.96 \%$ with LPN (7). A review study reported that RAP after PN occurred at mean 14.9 days, but since it may appear within 1-90 days, care should be taken for up to about 3 months after surgery (8). The etiology of RAP after PN is believed to be direct injury

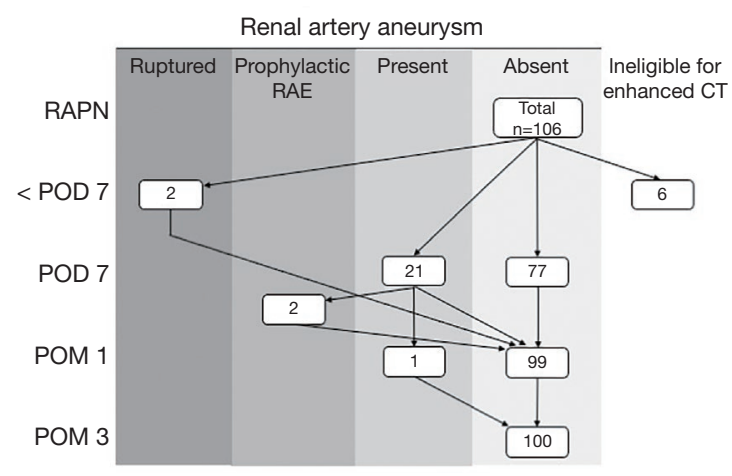

Figure 1 Clinical course of RAP in 106 patients. Among 106 patients who had undergone RAPN, 6 were excluded as ineligible for CE-CT. Ninety-eight completed routine CE-CT scans 7 days, 1 month and 3 months after RAPN, while 2 had emergency CE-CT scans. On POD7, 21 patients had RAPs, of which only 1 case remained at POM1. Within 1 week after routine CECT scans at 7 days, 2 patients underwent prophylactic RAE. No patient had RAPs at POM3. Abbreviations: RAP, renal artery pseudoaneurysm; RAPN, robot-assisted partial nephrectomy; CE-CT, contrast-enhanced computed tomography; RAE, renal artery selective embolization; POD, postoperative day; POM, postoperative month. to a segmental branch of the renal artery. Recent studies demonstrated incidence rates of asymptomatic unruptured RAP on contrast-enhanced computed tomography (CE-CT) after PN of $15.0 \%$ and $21.7 \%(9,10)$. Accordingly, the frequency of asymptomatic unruptured RAP is likely higher than expected, which would in turn indicate that RAP in RAPN has not been fully evaluated.

Here, we report the natural course of RAP by assessing postoperative renal condition with routine CE-CT after RAPN regardless of the presence or absence of clinical symptoms and factors affecting the occurrence of RAP.

We present the following article in accordance with the STROBE reporting checklist (available at https://dx.doi. org/10.21037/tau-21-384).

\section{Methods}

\section{Patient population}

From May 2016 to September 2020, 106 patients underwent RAPN for renal tumors at Jichi Medical University Hospital. As shown in Figure 1, six patients were excluded from the present analysis because they were ineligible for CE-CT due to asthma, allergy to contrast medium, or renal dysfunction. Among the 100 remaining patients, four underwent renal artery selective embolization (RAE). Two patients underwent emergency RAE due to ruptured RAP diagnosed by following the clinical course-the first with a decrease in blood pressure and progression of anemia 4 hours after RAPN and the second with fever 4 days after RAPN and a blood test showing anemia - and did not undergo routine CE-CT at 7 days after RAPN. The other two patients with RAP confirmed by routine CE-CT on the 7 days of RAPN underwent prophylactic RAE due to their social situation, namely difficulty in visiting the hospital if the RAP ruptured. Finally, in 98 patients examined for the clinical course of asymptomatic RAP managed by surveillance, excluding 2 who underwent emergency RAE, routine CE-CT was carried out 7 days, 1 month and 3 months after RAPN. Factors influencing the occurrence of RAP among these 98 patients, including 2 who underwent emergency RAE and excluding 2 who underwent prophylactic RAE, were analyzed by logistic regression analysis. Tumor and RAP size were determined by CT, and perioperative complications were graded by the Clavien-Dindo classification (11). This study was conducted in accordance with the Declaration of Helsinki (as revised in 2013). This study was approved by the Institutional Review Board of Jichi Medical University (A19-199) 
and withdrawal of consent to the use of medical data was by the opt-out system.

\section{Surgical technique}

All operations were performed using the da Vinci Si robotic platform (Intuitive Surgical, Inc., Sunnyvale, CA, USA). Six or seven ports were placed, of which two or three were for the assistant surgeons. Surgeries were performed by two surgeons (operator and assistant). The approach was chosen depending on the tumor location. The transperitoneal approach is generally used for lesions located anteriorly, while the retroperitoneal approach is used for lesions located posteriorly. In general, an extra arm was used. No ureteral catheters were used. An intraoperative ultrasonography probe (L43K: Hitachi, Tokyo, Japan) was used to confirm the margins of the tumor. Briefly, the renal hilum was dissected, allowing the clamping of individual renal arteries. The resection margin was delineated with ultrasound guidance. In the clamping method, bulldog clamps were used for total or partial renal artery clamping. After confirming that blood flow to the kidney was decreased by ultrasonography or by the renal parenchyma macroscopically turning from red to white, the tumor was bluntly dissected and enucleated and/or resected with a thin margin so as to preserve the normal parenchyma as much as possible. Some blood vessels flowing into and out of the tumor were sectioned after placement of hemolock clips by the assistant surgeon. The next procedure is parenchymal renorrhaphy (inner and outer layer sutures). The tumor bed was repaired with an inner running suture $(15 \mathrm{~cm} 3-0 \mathrm{~V}-\mathrm{Loc}$ 180 CV23: Covidien, New Haven, CT, USA); this repair was also conducted in cases in which the collecting ducts were released or renal sinus fat was exposed during tumor resection. After inner suturing, the clamps were removed (early unclamping). The outer continuous suture $(20 \mathrm{~cm}$ 2-0 V-Loc 180 GS21: Covidien, New Haven, CT, USA) with hemolock clips placed at exit points was then done while repeating the procedure of sliding the hemolock clips after tightening the suture as needed. For hemostasis, absorbable hemostats (largely SURGICEL; Johnson \& Johnson, Tokyo, Japan) were applied to the surface after renorrhaphy was completed.

\section{CT imaging and analysis}

\section{CT technique}

CT examinations were carried out using multi-detector row CT scanners, such as SOMATOM Definition Flash, SOMATOM Definition AS, and SOMATOM Sensation 64 (Siemens, Erlangen, Germany). Our institutional triphasic renal CT protocol consisted of acquisition of an unenhanced CT image of the upper abdomen, followed by a corticomedullary phase acquisition of the upper abdomen and a delayed nephrographic phase acquisition of the entire abdomen and pelvis. A fixed $100-$ or $120-k V p$ technique was used for all three phases, with automated tube current modulation and a variable tube current of 100-500 mA. Slice thickness is $2.5-3 \mathrm{~mm}$ in all phases. Patients were administered $540-600 \mathrm{mgI} / \mathrm{kg}$ of nonionic iodine contrast agent at a fixed intravenous injection rate of $2.5-3.0 \mathrm{~mL} / \mathrm{s}$ with the use of a power injector. The timing of the corticomedullary phase was established by bolus tracking (30-40 s after injection). The nephrographic phase image was acquired $80 \mathrm{~s}$ after the corticomedullary phase image acquisition (110-120 s after injection).

\section{CT image evaluation}

All acquired images were transferred to our clinicallyused server and evaluated with a picture archiving and communication system (PACS; Synapse, Fujifilm Medical Systems, Tokyo, Japan) with 2-megapixel high resolution liquid crystal display. A single experienced diagnostic radiologist who had no knowledge of the surgical or clinical results reviewed axial and coronal multiplanar reformatted images $(3 \mathrm{~mm})$ of multiphase images, and assessed vascular complications, such as RAP, irregularity of renal arteries and contrast extravasation. RAP was defined as a saccularor fusiform-shaped dilatation of the renal arterial branches compared with the surrounding normal renal arterial branches. Potential RAP smaller than $2 \mathrm{~mm}$ in diameter were excluded because of imaging limitations, such as slicerelated pseudolesion or the possibility of renal artery stump dilation.

\section{Statistical analysis}

Categorical variables were expressed as frequencies and percentages, and differences were compared using the chi-square test and Mann-Whitney $U$ test. Univariate analyses for the occurrence of RAPs were performed by logistic regression methods. Subsequent multivariate logistic regression analysis for the occurrence of RAPs was performed with factors whose $\mathrm{P}$ values greater than 0.1 in the univariate analysis. In the multivariate analysis, the inner suture time, which is a component of the renorrhaphy time, 
was included in the warm ischemic time, so warm ischemic time was used as representative. All $\mathrm{P}$ values presented are two-sided. Statistical significance was calculated using StatView ver. 5 (Abacus Concepts, CA, USA), with $\mathrm{P}$ values of $<0.05$ considered to indicate statistical significance.

\section{Results}

Patient characteristics and perioperative outcomes are detailed in Table 1. A total of 2 operators and 8 assistants were involved in the surgery. There was no significant difference in the chi-square test between the operator (A vs. B) or surgical approach (transperitoneal vs. retroperitoneal) $(\mathrm{P}=0.1248)$ (data not shown). No patient required intraoperative blood transfusion or was converted to open surgery and nephrectomy. Urinary leakage was not observed. A positive surgical margin was found in one patient (1.0\%) with chromophobe renal cell carcinoma (RCC). No patients, except those who underwent emergency RAE, experienced any signs of potential pseudoaneurysm, such as gross hematuria. A major complication of Clavien-Dindo grade 3 or higher was found in 4 patients with RAE. RAE was the only procedure with a Clavien-Dindo grade 3 or higher complication.

Figure 1 outlines the clinical course of RAPs. CE-CT detected 28 RAPs in 23 (23.0\%) of 100 patients by 7 days after RAPN and routine CE-CT detected 25 RAPs in 21 (21.4\%) of 98 patients (excluding 2 patients who underwent emergency RAE by 7 days after RAPN due to inability to measure the exact size of RAP) on 7 days of RAPN. By timing, $21(21.4 \%), 1(1.0 \%)$, and $0(0 \%)$ of patients with RAP were diagnosed by routine CE-CT at 7 days, 1 month, and 3 months after RAPN, respectively. The median maximum diameter of RAP was $6.6 \mathrm{~mm}$ (interquartile range $5.2 \mathrm{~mm}$; range, $3.0-16.0 \mathrm{~mm}$ ). All of the RAPs were located on the interlobar, arcuate or interlobular arteries. The RAP sizes of the two patients who underwent prophylactic TAE were 12 and $16 \mathrm{~mm}$, respectively. Five RAPs $(12.4,13.3,14.3,15.0$, and $15.2 \mathrm{~mm}$ in diameter) with a size larger than $12 \mathrm{~mm}$ and smaller than $16 \mathrm{~mm}$ observed in 5 patients at 7 days after RAPN resolved spontaneously 1 month after RAPN. Among all patients, only one patient had RAP 1 month after RAPN, with an RAP size course of 4.8, 3.7, and $0 \mathrm{~mm}$ at 7 days, 1 month, and 3 months after RAPN, respectively. As shown in Figure 2, one patient with three RAPs (10.9 mm in Figure 2A, $14.3 \mathrm{~mm}$ in Figure 2B, and $6.0 \mathrm{~mm}$ in Figure $2 C$, as shown by a white arrow with a black border) found 7 days after RAPN resolved by 1 month after RAPN.

Table 2 shows patient characteristics and perioperative outcomes classified by the presence or absence of RAP managed by surveillance. Age ( $\mathrm{P}=0.0325)$, R.E.N.A.L [radius (tumor size as maximal diameter), exophytic/endophytic properties of tumor, nearness of tumor deepest portion to collecting system or sinus, anterior/posterior descriptor and location relative to polar line] nephrometry score $(\mathrm{P}=0.0410)$, warm ischemic time $(\mathrm{P}=0.0230)$, renorrhaphy time $(\mathrm{P}=0.0103)$, and blood loss $(\mathrm{P}=0.0128)$ were significantly associated with the presence or absence of RAP.

Table 3 shows factors influencing the occurrence of RAP on logistic regression analysis using the five factors related to the presence or absence of RAP shown in Table 2. First, logistic regression analysis was performed on the five factors as continuous variables. In univariate analysis, R.E.N.A.L nephrometry score [odds ratio $(\mathrm{OR}) 1.374, \mathrm{P}=0.0382$ ], warm ischemic time (OR 1.085, $\mathrm{P}=0.0393)$, and renorrhaphy (inner and outer layer suture) time (OR 1.055, P=0.0408) were significantly associated with the development of RAP. In multivariate analysis using these three factors plus additional two factors with $\mathrm{P}$ values $<0.1$ in univariate analysis—age (OR 0.963, $\mathrm{P}=0.0617)$ and blood loss (OR 1.006, $\mathrm{P}=0.0715)$ - no significant factors were detected, but age showed a tendency to an association $(\mathrm{P}=0.0643)$. Therefore, a logistic regression univariate analysis was performed with classification by a median age of 63 years, which showed that younger age was a significant factor in the occurrence of RAP (OR 4.267, $\mathrm{P}=0.0098$ ). Furthermore, on classification into three groups such that the number of cases in each group was almost equal, youngest age was significantly associated with the occurrence of RAP in both logistic regression univariate $(\mathrm{OR} 0.144, \mathrm{P}=0.0179)$ and multivariate analysis (OR 0.124, $\mathrm{P}=0.0148)$.

\section{Discussion}

In this study, we found that RAPs occurring after RAPN resolved spontaneously after 3 months, and showed no recurrence at a median follow-up period of 21.6 months. We also found that the occurrence of RAP may associated with a patient age younger than the predominant age of renal cancer, namely under age 56 years.

Pseudoaneurysm can arise from various arteries and is an undesirable complication after treatment of various organs and disorders. Although the cause has not been elucidated, or such characteristics as whether it occurs only in certain persons or in persons who originally 
Table 1 Patient characteristics and perioperative outcomes

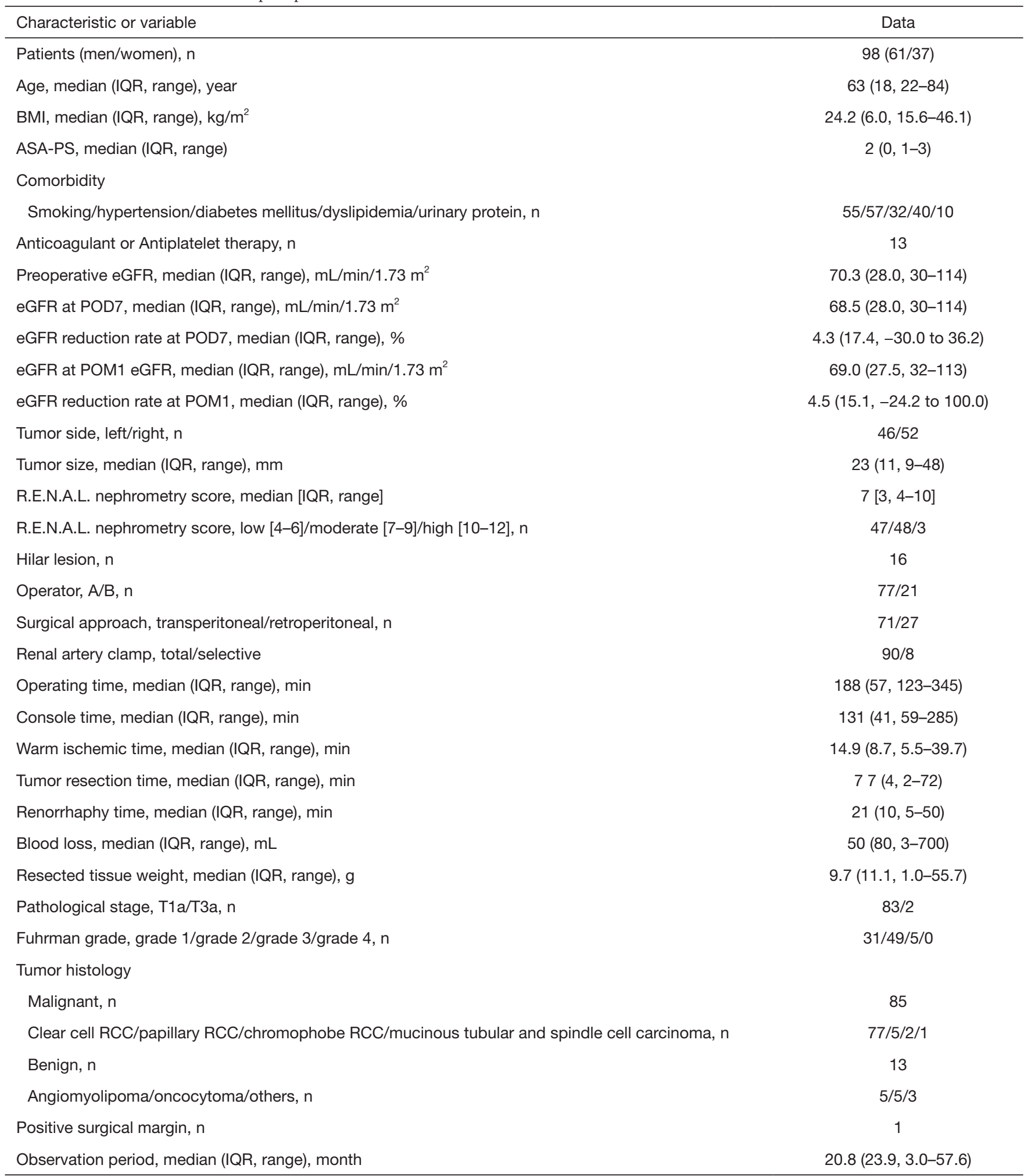

IQR, interquartile range; BMI, body mass index; ASA-PS, American Society of Anesthesiologists physical status; eGFR, estimated glomerular filtration rate; R.E.N.A.L., radius (tumor size as maximal diameter), exophytic/endophytic properties of tumor, nearness of tumor deepest portion to collecting system or sinus, anterior/posterior descriptor and location relative to polar line; RCC, renal cell carcinoma. 


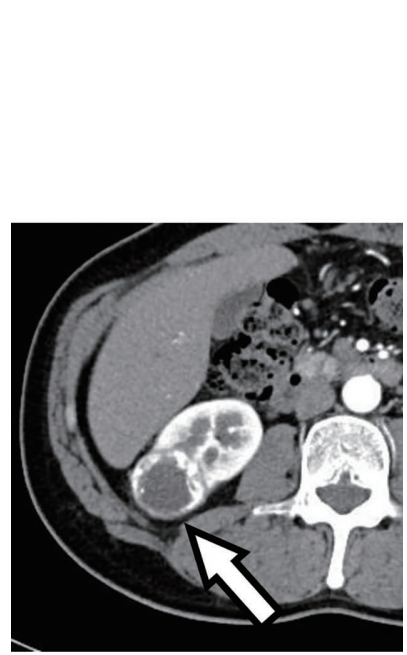

Preoperative image
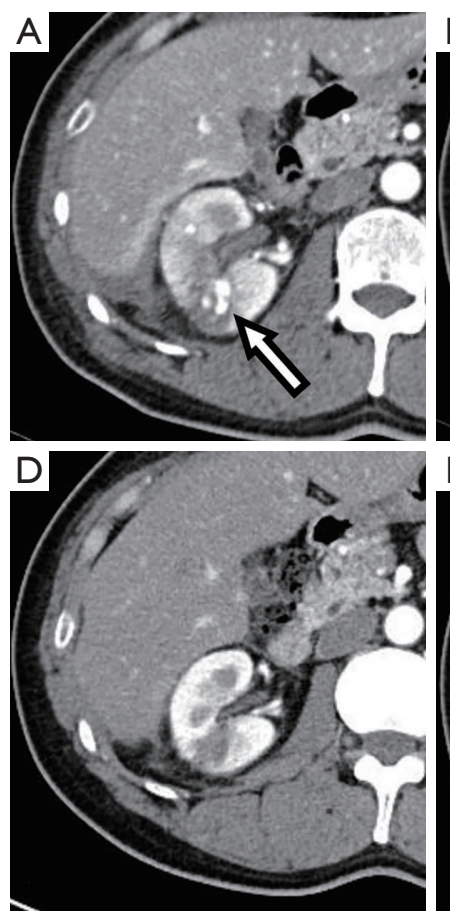
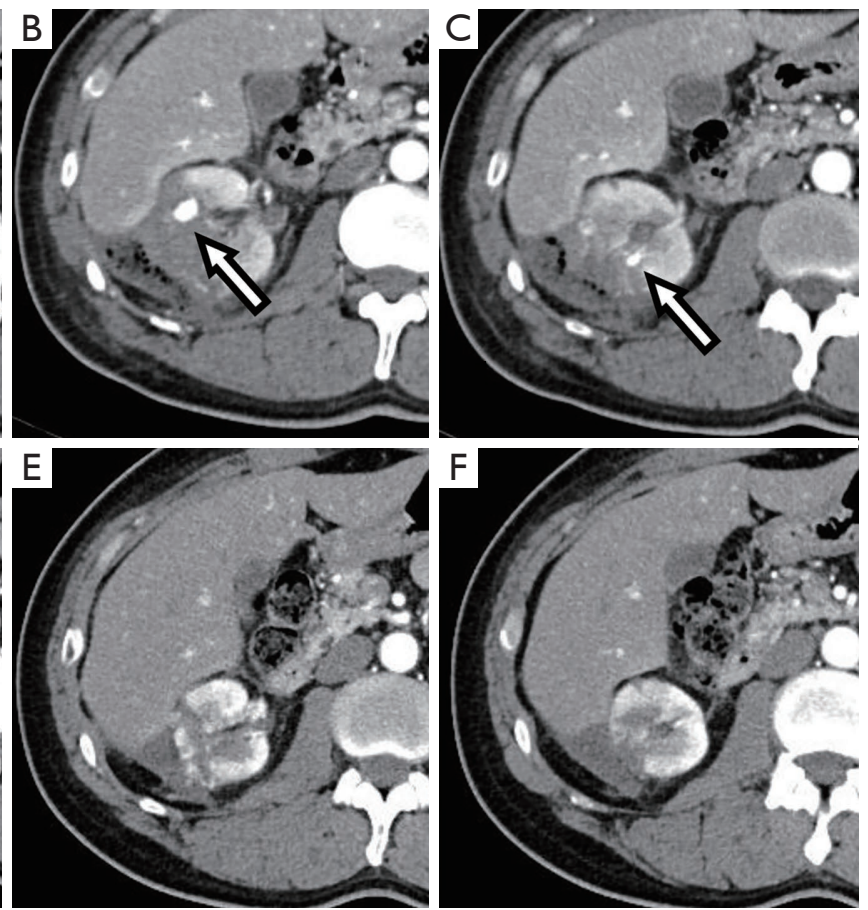

Figure 2 Contrast enhanced CT images. (A-C) Arterial phase CT images on POD7 after RAPN show multiple nodular enhancements that suggest RAPs. (D-F) Arterial phase CT images on POM1 show disappearance of RAPs. All three RAPs [10.9 mm in (A), $14.3 \mathrm{~mm}$ in (B) and $6.0 \mathrm{~mm}$ in $(\mathrm{C})$; shown by a white arrow with a black border] found on POD7 after RAPN had resolved by 1 month after RAPN as shown in (D-F). Abbreviations: CT, computed tomography; POD, postoperative day; RAPN, robot-assisted partial nephrectomy; RAP, renal artery aneurysm; POM, postoperative month.

Table 2 Patient characteristics and perioperative outcomes by the presence or absence of RAP

\begin{tabular}{|c|c|c|c|}
\hline Characteristics or variables & \multicolumn{2}{|c|}{ RAP } & $P$ value \\
\hline Men/women, $\mathrm{n}$ & $13 / 8$ & $48 / 29$ & $0.9711^{\dagger}$ \\
\hline Age, median (IQR, range), year & $56(8.8,22-77)$ & $65(17.5,35-84)$ & $0.0325^{\dagger+}$ \\
\hline BMI, median (IQR, range), $\mathrm{kg} / \mathrm{m}^{2}$ & $25.6(5.9,15.6-39.4)$ & $24.2(6.4,16.6-46.1)$ & $0.3985^{\dagger+}$ \\
\hline \multicolumn{4}{|l|}{ Comorbidity, +/-, n } \\
\hline Smoking & $8 / 13$ & $47 / 30$ & $0.0604^{\dagger}$ \\
\hline Hypertension & $12 / 9$ & $45 / 32$ & $0.9148^{\dagger}$ \\
\hline Diabetes mellitus & $5 / 16$ & $27 / 50$ & $0.3296^{\dagger}$ \\
\hline Anticoagulant or antiplatelet therapy, $+/-, \mathrm{n}$ & $2 / 19$ & $11 / 66$ & $0.5685^{\dagger}$ \\
\hline Preoperative eGFR, median (IQR, range), $\mathrm{mL} / \mathrm{min} / 1.73 \mathrm{~m}^{2}$ & $70.0(24.8,44.0-114.0)$ & $70.6(31.0,30.0-113.0)$ & $0.4644^{\dagger \dagger}$ \\
\hline
\end{tabular}

Table 2 (continued) 
Table 2 (continued)

\begin{tabular}{|c|c|c|c|}
\hline Characteristics or variables & \multicolumn{2}{|c|}{ RAP } & $P$ value \\
\hline eGFR at POD7, median (IQR, range), $\mathrm{mL} / \mathrm{min} / 1.73 \mathrm{~m}^{2}$ & $68(22.0,48-114)$ & $69(29.0,30-114)$ & $0.9379^{\dagger+}$ \\
\hline eGFR reduction rate at POD7, median (IQR, range), \% & $6.6(17.8,-15.2$ to 27.6$)$ & $4.1(17.3,-30.0$ to 36.1$)$ & $0.1673^{\dagger \dagger}$ \\
\hline eGFR at POM1 eGFR, median (IQR, range), $\mathrm{mL} / \mathrm{min} / 1.73 \mathrm{~m}^{2}$ & $71.1(31.8,38.0-93.0)$ & $69.0(27.0,32.0-113.0)$ & $0.4170^{\dagger+}$ \\
\hline Tumor side, left/right, $\mathrm{n}$ & $9 / 12$ & $37 / 40$ & $0.2603^{\dagger}$ \\
\hline Tumor size, median (IQR, range), mm & $2.1(0.9,0.9-4.3)$ & $2.4(1.2,1.0-4.8)$ & $0.6724^{\dagger \dagger}$ \\
\hline R.E.N.A.L. nephrometry score, median [IQR, range] & $8[3,4-10]$ & $6[3,4-10]$ & $0.0410^{\dagger+}$ \\
\hline R.E.N.A.L. nephrometry score, low [4-6]/moderate [7-9]/high [10-12], n & $6 / 13 / 2$ & $41 / 35 / 1$ & $0.0359^{\dagger}$ \\
\hline Surgical approach, transperitoneal/retroperitoneal, $\mathrm{n}$ & $13 / 8$ & $58 / 19$ & $0.2224^{\dagger}$ \\
\hline Renal artery clamp, total/selective & $20 / 1$ & $70 / 7$ & $0.5207^{\dagger}$ \\
\hline Collection system opening, $+/-, \mathrm{n}$ & $5 / 16$ & $9 / 68$ & $0.1594^{\dagger}$ \\
\hline Renal sinus exposure, $+/-, \mathrm{n}$ & $9 / 12$ & $29 / 48$ & $0.6650^{\dagger}$ \\
\hline Operating time, median (IQR, range), min & $188(45.0,154-266)$ & $188(63.8,123-345)$ & $0.4232^{\dagger+}$ \\
\hline Console time, median (IQR, range), min & $139(24.8,64-211)$ & $130(53.5,59-285)$ & $0.4777^{\dagger \dagger}$ \\
\hline Warm ischemic time, median (IQR, range), min & $20.0(9.6,7.8-29.1)$ & $14.4(7.2,5.5-39.7)$ & $0.0230^{\dagger+}$ \\
\hline Tumor resection time, median [IQR, range], min & $9[3,3-17]$ & $7[4,2-72]$ & $0.0525^{\dagger \dagger}$ \\
\hline Observation period, median (IQR, range), month & $22.6(24.6,4.9-48.1)$ & $20.5(22.1,3.0-57.4)$ & $0.8727^{\dagger \dagger}$ \\
\hline
\end{tabular}

${ }^{\dagger}$, Chi-square test; ${ }^{\dagger \dagger}$, Mann-Whitney U test. RAP, renal artery pseudoaneurysm; IQR, interquartile range; BMI, body mass index; ASA-PS, American Society of Anesthesiologists physical status; eGFR, estimated glomerular filtration rate; R.E.N.A.L., radius (tumor size as maximal diameter), exophytic/endophytic properties of tumor, nearness of tumor deepest portion to collecting system or sinus, anterior/ posterior descriptor and location relative to polar line; RCC, renal cell carcinoma.

have a vascular lesion (12), and how often it disappears spontaneously, it is believed to commonly occur during surgical vascular injury and its healing process. Regarding the etiology of RAP after PN, Singh and Gill proposed two mechanisms: (I) inadvertent vascular injury during tumor resection, and (II) vascular injury during parenchymal renorrhaphy (13). Previous reports demonstrated that the retroperitoneal approach, tumors buried in the renal parenchyma, tumor complexity, and renal sinus exposure affected the development of RAP, suggesting that treating deep kidneys with tumor complexity within a narrow surgical field increases the risk of $\operatorname{RAP}(7,14,15)$. In our study, surgical approach and renal sinus exposure did not affect the presence or absence of RAP (Table 2). In contrast, high R.E.N.A.L. nephrometry scores, which indicate tumor complexity, affected the presence or absence of RAP 
Table 3 Factors influencing the occurrence of RAP by logistic regression analysis

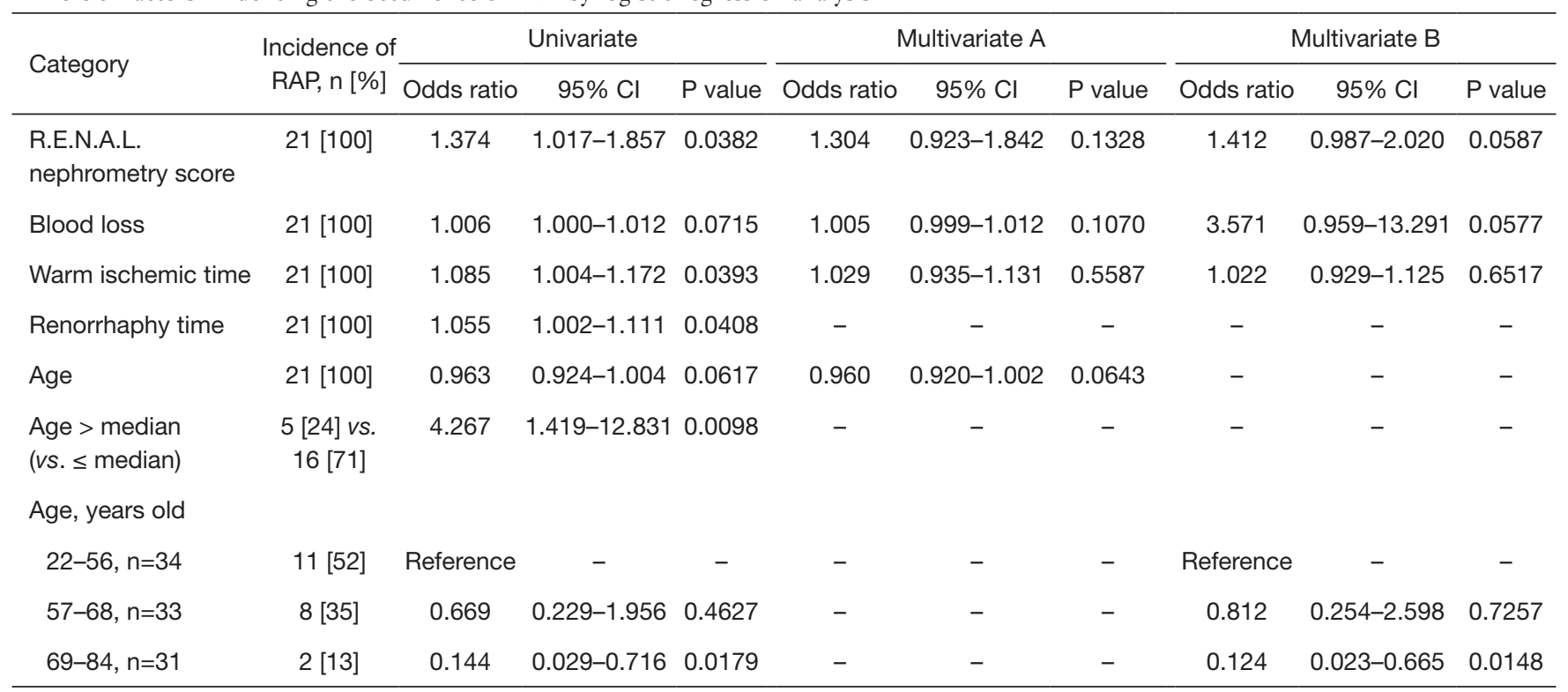

In the multivariate analyses, the inner suture time, which is a component of renorrhaphy time, is included in the warm ischemic time, so warm ischemic time was used as a representative. Multivariate A is a multivariate analysis in which factors are used as continuous variables, while multivariate $B$ is a multivariate analysis in which only the factor of age is categorized. Cl, confidence interval; RAP, renal artery pseudoaneurysm; BMI, body mass index; eGFR, estimated glomerular filtration rate; R.E.N.A.L., radius (tumor size as maximal diameter), exophytic/endophytic properties of tumor, nearness of tumor deepest portion to collecting system or sinus, anterior/posterior descriptor and location relative to polar line; RCC, renal cell carcinoma.

(Table 2) and were a significant factor in the occurrence of RAP (Table 3). The same tendency was seen when only the "E" component of the R.E.N.A.L. nephrometry scores was examined (data not shown). Supporting the vascular injury hypothesis, previous reports have shown that early unclamping might reduce the risk of RAP after RAPN or LPN $(14,16,17)$. Kondo et al. stated that early unclamping can provide information regarding arterial bleeding from the resected bed before renal parenchymal suture. As arterial bleeding from the resected bed is controlled by the inner suture rather than the renal parenchymal suture, additional inner suturing should provide hemostasis of arterial bleeding with early unclamping (16). Early unclamping was also adopted, but the incidence of RAP $(23 \%)$ was higher than that reported by Kondo et al. (11\%) (16). The present study is a review of all cases from the start of RAPN at our university hospital; this difference in outcomes may be related to technical proficiency in renorrhaphy, followed by the significant association of longer renorrhaphy time with both the presence or absence of RAP and the occurrence of this complication (Tables 2,3). In addition, with regard to OPN, Ota et al. reported the safety and effectiveness of the soft-coagulation system for
PN without parenchymal renorrhaphy, excluding hilar tumors (18). The soft coagulation system of the VIO 300D was used with the effect level set at 7 and output of $80 \mathrm{~W}$, using a ball-type electrode to increase the contact area with the resection bed. Tachibana et al. also showed that the non-renorrhaphy (inner layer sutures only) technique using the soft-coagulation system may produce a lower risk of RAP than renorrhaphy (inner and outer layer sutures) (19). Similarly, for RAPN in patients with cT1a renal tumors, RAP did not occur with hemostasis by soft coagulation only, namely with inner sutures and without outer sutures $(20,21)$. Thus, the soft coagulation system may help prevent the development of RAP occurring following surgical resection.

In contrast, uterine arterial pseudoaneurysm occurs rarely following cesarean section, and may also occur due to factors other than surgical procedures (22). It is noteworthy that the target demographic for this condition is young fertile women. Moreover, lower age ( $<65.5$ years) was reported to be an independent risk factor for the development of pseudoaneurysm after pancreaticoduodenectomy, while a systematic review reported a higher number of cases of ankle pseudoaneurysm among young adults, namely in 15 (65\%) of 23 case reports 
published from 1966 until $2018(23,24)$. On the contrary, while a positive effect of aging on the occurrence of RAP after RAPN has been reported, little discussion of this association has appeared (16). In logistic regression analysis of the factors that influenced the occurrence of RAP in our study (Table 2), young population and factors involved in surgery such as tumor resection time, renorrhaphy (inner and outer layer sutures) time, blood loss, and R.E.N.A.L. nephrometry scores were significant factors in univariate analysis. In addition, there was no difference among patients in baseline characteristics by age (data not shown). These results support the hypothesis of RAP occurrence. In multivariate analysis, in contrast, the only significant factor was a young population age. Angiogenesis plays an important role in the repair of blood vessels and tissues, and the impairment of this process in older patients has been demonstrated $(25,26)$. Taken together, younger age may contribute to the occurrence of RAP. A conclusive answer to the possible role of age in RAP will require evaluation in a larger number of patients and basic research on angiogenic factors.

As shown in Figure 1, the most impactful result of our study is that, regardless of cause, pseudoaneurysms of approximately $15 \mathrm{~mm}$ resolved spontaneously after 3 months, and showed no recurrence with a median observation period of 20.8 months. Accordingly, our results suggest indicators of causation and resolution in the natural history of RAP, namely potential target values for RAP size and observation period. The size of RAP at which resolution occur is consistent with the size reported for the spontaneous disappearance of uterine arterial pseudoaneurysm (27). In any case, our findings suggest that one method of preventing RAP might be hemostasis of the resected surface by soft coagulation with an inner layer suture $(20,21)$. RAP is generally described as occurring about 2 weeks after surgery, but may occur anywhere up to 6 months after LPN or RAPN or 2 or more years after OPN, and long-term follow-up should therefore not be neglected $(28,29)$. In addition, although routine postoperative CE-CT is harmful in terms of renal function, radiation exposure and cost, Kondo et al. have pointed out that the benefits can outweigh the harm given the complications and costs which occur after RAP rupture (16). Conversely, routine postoperative CE-CT may carry greater risk of overimaging, overdiagnosis, and overtreatment of asymptomatic RAP, as in our two patients who underwent prophylactic RAE for asymptomatic RAP diagnosed by routine CE-CT 7 days after RAPN. Further, the usefulness of Doppler ultrasonography for the early detection of significant asymptomatic RAP has been reported (30). Against this background, we found that RAPs of $15 \mathrm{~mm}$ or less disappear spontaneously after 3 months, suggesting that routine early postoperative imaging including CE-CT is not required in the absence of clinical signs or symptoms such as gross hematuria.

This study has some limitations. First, it was a retrospective study with a small sample size at a single university hospital, hindering generalization of the results. Second, as many surgeons were involved as assistants, it was not possible to examine the effect of their proficiency in surgical techniques. Third, diagnosis of RAP was performed by a single radiologist, albeit one that was skilled in this procedure. Fourth, patients with renal impairment, allergy to contrast medium, or asthma were excluded from this study. Fifth, this study was initiated at a single university hospital in a situation where the natural history of RAP was unknown, and possible complications other than RAP were identified. We therefore planned to carefully follow the course of all consecutive patients and perform routine CE$\mathrm{CT}$ without missing any cases. Patients who understood the above underwent RAPN, resulting in overimaging. Sixth, although age was detected as a factor in the development of RAP, we were unable to evaluate the mechanism of this effect. We are currently preparing basic research into this question. Further progress in this field awaits a larger multicenter, prospective, randomized study.

In conclusion, RAPs generated after RAPN were followed by routine CE-CT. Asymptomatic RAP up to $15 \mathrm{~mm}$ in diameter resolved spontaneously within 3 months after RAPN. Young age (under 56 years) may be a factor in the development of RAP. Although further research is needed on factors such as long-term follow-up and age, we suggest that current efforts to prevent RAP should consider the use of soft coagulation in the repair of resected surfaces.

\section{Acknowledgments}

The authors received no funding for this study. We thank Guy Harris D.O. from DMC Corp. (www.dmed.co.jp) for editing drafts of this manuscript.

Funding: None.

\section{Footnote}

Reporting Checklist: The authors have completed the STROBE reporting checklist. Available at https://dx.doi. 
org/10.21037/tau-21-384

Data Sharing Statement: Available at https://dx.doi. org/10.21037/tau-21-384

Peer Review File: Available at https://dx.doi.org/10.21037/ tau-21-384

Conflicts of Interest: All authors have completed the ICMJE uniform disclosure form (available at https://dx.doi. org/10.21037/tau-21-384). The authors have no conflicts of interest to declare.

Ethical Statement: The authors are accountable for all aspects of the work in ensuring that questions related to the accuracy or integrity of any part of the work are appropriately investigated and resolved. This study was conducted in accordance with the Declaration of Helsinki (as revised in 2013). This study was approved by the Institutional Review Board of Jichi Medical University (A19-199) and withdrawal of consent to the use of medical data was by the opt-out system.

Open Access Statement: This is an Open Access article distributed in accordance with the Creative Commons Attribution-NonCommercial-NoDerivs 4.0 International License (CC BY-NC-ND 4.0), which permits the noncommercial replication and distribution of the article with the strict proviso that no changes or edits are made and the original work is properly cited (including links to both the formal publication through the relevant DOI and the license). See: https://creativecommons.org/licenses/by-nc-nd/4.0/.

\section{References}

1. Huang WC, Elkin EB, Levey AS, et al. Partial nephrectomy versus radical nephrectomy in patients with small renal tumors--is there a difference in mortality and cardiovascular outcomes? J Urol 2009;181:55-61; discussion 61-2.

2. Thompson RH, Boorjian SA, Lohse CM, et al. Radical nephrectomy for $\mathrm{p}$ T1a renal masses may be associated with decreased overall survival compared with partial nephrectomy. J Urol 2008;179:468-71; discussion 472-3.

3. Yamazaki M, Takayama T, Fujisaki A, et al. Robotassisted partial nephrectomy of initial cases using a 3D square-block type kidney model. Transl Androl Urol 2020;9:494-500.
4. Kwon Kim J, Ryu H, Kim M, et al. Personalised threedimensional printed transparent kidney model for robotassisted partial nephrectomy in patients with complex renal tumours (R.E.N.A.L. nephrometry score $\geq 7$ ): a prospective case-matched study. BJU Int 2021;127:567-74.

5. Fujisaki A, Takayama T, Yamazaki M, et al. Utilization of a three-dimensional printed kidney model for favorable TRIFECTA achievement in early experience of robotassisted partial nephrectomy. Transl Androl Urol 2020;9:2697-704.

6. Ngo TC, Lee JJ, Gonzalgo ML. Renal pseudoaneurysm: an overview. Nat Rev Urol 2010;7:619-25.

7. Gill IS, Kavoussi LR, Lane BR, et al. Comparison of 1,800 laparoscopic and open partial nephrectomies for single renal tumors. J Urol 2007;178:41-6.

8. Jain S, Nyirenda T, Yates J, et al. Incidence of renal artery pseudoaneurysm following open and minimally invasive partial nephrectomy: a systematic review and comparative analysis. J Urol 2013;189:1643-8.

9. Takagi T, Kondo T, Tajima T, et al. Enhanced computed tomography after partial nephrectomy in early postoperative period to detect asymptomatic renal artery pseudoaneurysm. Int J Urol 2014;21:880-5.

10. Omae K, Kondo T, Takagi T, et al. Renal sinus exposure as an independent factor predicting asymptomatic unruptured pseudoaneurysm formation detected in the early postoperative period after minimally invasive partial nephrectomy. Int J Urol 2015;22:356-61.

11. Dindo D, Demartines N, Clavien PA. Classification of surgical complications: a new proposal with evaluation in a cohort of 6336 patients and results of a survey. Ann Surg 2004;240:205-13.

12. Matsubara S, Nonaka H, Kobayashi M, et al. Uterine artery pseudoaneurysm after dilation and curettage in a woman with multiple hepatic and pulmonary cavernous hemangiomas. Int J Gynaecol Obstet 2014;125:84-5.

13. Singh D, Gill IS. Renal artery pseudoaneurysm following laparoscopic partial nephrectomy. J Urol 2005;174:2256-9.

14. Chung DY, Lee JS, Ahmad A, et al. Lessons learned from clinical outcome and tumor features of patients underwent selective artery embolization due to postoperative bleeding following 2076 partial nephrectomies: propensity scoring matched study. World J Urol 2020;38:1235-42.

15. Kriegmair MC, Mandel P, Rathmann N, et al. Open Partial Nephrectomy for High-Risk Renal Masses Is Associated with Renal Pseudoaneurysms: Assessment of a Severe Procedure-Related Complication. Biomed Res Int 2015;2015:981251. 
16. Kondo T, Takagi T, Morita S, et al. Early unclamping might reduce the risk of renal artery pseudoaneurysm after robot-assisted laparoscopic partial nephrectomy. Int J Urol 2015;22:1096-102.

17. Nguyen MM, Gill IS. Halving ischemia time during laparoscopic partial nephrectomy. J Urol 2008;179:627-32; discussion 632.

18. Ota T, Komori H, Rii J, et al. Soft coagulation in partial nephrectomy without renorrhaphy: feasibility of a new technique and early outcomes. Int J Urol 2014;21:244-7.

19. Tachibana H, Takagi T, Kondo T, et al. Comparison of perioperative outcomes with or without renorrhaphy during open partial nephrectomy: A propensity scorematched analysis. Int Braz J Urol 2018;44:467-74.

20. Tohi Y, Murata S, Makita N, et al. Comparison of perioperative outcomes of robot-assisted partial nephrectomy without renorrhaphy: Comparative outcomes of cT1 versus cT1b renal tumors. Int J Urol 2019;26:885-9.

21. Tohi Y, Murata S, Makita N, et al. Absence of asymptomatic unruptured renal artery pseudoaneurysm on contrast-enhanced computed tomography after robot-assisted partial nephrectomy without parenchymal renorrhaphy. Asian J Urol 2020;7:24-8.

22. Baba Y, Takahashi H, Ohkuchi A, et al. Uterine artery pseudoaneurysm: its occurrence after non-traumatic events, and possibility of "without embolization" strategy. Eur J Obstet Gynecol Reprod Biol 2016;205:72-8.

23. Chipaila J, Kato H, lizawa Y, et al. Prolonged operating

Cite this article as: Takayama T, Fujita A, Sugihara T, Fujisaki A, Yamazaki M, Kameda T, Kamei J, Ando S, Kurokawa S, Fujimura T. Natural history of asymptomatic renal artery pseudoaneurysm after robot-assisted partial nephrectomy. Transl Androl Urol 2021;10(9):3555-3565. doi: 10.21037/tau-21-384 time is a significant perioperative risk factor for arterial pseudoaneurysm formation and patient death following hemorrhage after pancreaticoduodenectomy. Pancreatology 2020;20:1540-9.

24. Aimanan K, Lim SY, Mohd Nor MR, et al. Systemic review of global case reports on ankle pseudo aneurysm: Analysis of epidemiology, clinical presentation, diagnosis and treatment. Med J Malaysia 2020;75:88-93.

25. Fan Y, Arif A, Gong Y, et al. Stimulus-dependent phosphorylation of profilin-1 in angiogenesis. Nat Cell Biol 2012;14:1046-56.

26. Moriya J, Minamino T. Angiogenesis, Cancer, and Vascular Aging. Front Cardiovasc Med 2017;4:65.

27. Takahashi H, Baba Y, Usui R, et al. Spontaneous resolution of post-delivery or post-abortion uterine artery pseudoaneurysm: A report of three cases. J Obstet Gynaecol Res 2016;42:730-3.

28. Otaño-Rivera V, Chelluri R, Guzzo TJ, et al. Detection of renal artery pseudo-aneurysm six months after robotic assisted partial nephrectomy in a patient whose only complaint was flank pain. Urol Case Rep 2020;33:101259.

29. Crestani A, Rossanese M, Sponza M, et al. Late Arteriovenous Fistula After Partial Nephrectomy in Solitary Kidney. J Endourol Case Rep 2019;5:81-3.

30. Nouhaud FX, Williams M, Arnfield E, et al. Is postoperative Doppler ultrasonography useful for the early detection of asymptomatic pseudoaneurysm and prevention of haemorrhagic complications after partial nephrectomy? BJU Int 2018;122 Suppl 5:15-21. 